\title{
Reversal of Experimental Autoimmune Encephalomyelitis with a Hydroxamate Inhibitor of Matrix Metalloproteases
}

\author{
Koenraad Gijbels, * Richard E. Galardy ${ }^{\ddagger}$ and Lawrence Steinman* \\ *Department of Neurology and Neurological Sciences, Stanford University Medical School, Stanford, California 94305; \\ and ${ }^{\ddagger}$ Glycomed Inc., Alameda, California 94501
}

\begin{abstract}
Gelatinases, belonging to the matrix metalloproteases, contribute to tissue destruction in inflammatory demyelinating disorders of the central nervous system such as multiple sclerosis. We used experimental autoimmune encephalomyelitis (EAE) as an animal model to evaluate the effect of a hydroxamate matrix metalloprotease inhibitor (GM 6001) on inflammatory demyelination. A single dose of the inhibitor, given intraperitoneally, provided sufficient levels in the cerebrospinal fluid of animals with EAE to induce at least a partial inhibition of the gelatinase activity in the cerebrospinal fluid. When administered daily either from the time of disease induction or from the onset of clinical signs, GM 6001 suppressed the development or reversed clinical EAE in a dose-dependent way, respectively. Animals returned to the same clinical course as the nontreated group after cessation of treatment. Animals treated from the onset of clinical signs had normal permeability of the blood-brain barrier, compared with the enhanced permeability in nontreated animals. These results indicate that matrix metalloprotease inhibition can reverse ongoing EAE. This effect appears to be mediated mainly through restoration of the damaged blood-brain barrier in the inflammatory phase of the disease, since the degree of demyelination and inflammation did not differ between the treatment groups. ( $J$. Clin. Invest. 1994. 94:2177-2182.) Key words: multiple sclerosis • demyelinating diseases - protease inhibitors - inflammation • blood-brain barrier
\end{abstract}

\section{Introduction}

Multiple sclerosis (MS $)^{1}$ is an inflammatory demyelinating disorder of the central nervous system (CNS) (1). MS is characterized histopathologically by focal lesions in different stages of evolution in the white matter of the CNS. Breakdown of the blood-brain barrier and inflammatory perivascular infiltration

Address correspondence to K. Gijbels, Department of Neurology and Neurological Sciences, Beckman Center, Room B002, Stanford University Medical Center, Stanford, CA 94305-5429. 1994.

Received for publication 16 May 1994 and in revised form 27 July

1. Abbreviations used in this paper: CNS, central nervous system; CSF, cerebrospinal fluid; EAE, experimental autoimmune encephalomyelitis; MMP, matrix metalloprotease; MS, multiple sclerosis.

J. Clin. Invest.

(C) The American Society for Clinical Investigation, Inc. 0021-9738/94/12/2177/06 \$2.00

Volume 94, December 1994, 2177-2182 are the first events in lesion formation and are followed by demyelination and astrogliosis. Local inflammation is most probably induced by an autoimmune response against the myelin sheath (2). Proteolytic enzymes are believed to contribute to the inflammatory tissue damage in this disease (3). We demonstrated the presence of gelatinolytic activity in the cerebrospinal fluid (CSF) of patients with MS (4) and in the CSF of mice with experimental autoimmune encephalomyelitis (EAE), an animal model for MS ( 5 ). We identified this gelatinolytic activity as gelatinase (type IV collagenase), belonging to the family of the matrix metalloproteases (MMPs) (6), based on the molecular weight and the inhibition by protease inhibitors. Both gelatinase A and B were detectable in the CSF, but levels of gelatinase $\mathrm{B}$ were more elevated in inflammatory CNS disorders. We recently confirmed the identity of gelatinase $B$ in the CSF by Western blot analysis (unpublished observation). Gelatinases degrade type IV collagen one of the main components of the basement membrane surrounding the capillaries (7). Direct injection of gelatinase into the brain causes disruption of the basement membrane and is associated with breakdown of the blood-brain barrier (8). Myelin basic protein, one of the major protein components of the myelin membrane, is digested in vitro by gelatinase $B(5)$. We recently demonstrated the presence of gelatinase B in MS lesions in inflammatory cells close to the capillary basement membrane as well as in macrophages at the demyelinating edge of the lesion (9). This indicates that at least one of the MMPs, gelatinase B, plays an important role in two key processes in the pathophysiology of MS: breakdown of the blood-brain barrier and demyelination.

Although the concerted action of several proteases as well as other inflammatory mediators is most probably responsible for the pathophysiological process in these disorders, inhibition of one component could be sufficient to halt this process. Inhibition of MMP activity was demonstrated to be beneficial in models of other inflammatory disorders, where MMPs are considered to be of pathological importance, such as rheumatoid arthritis (10). The same MMP inhibitor that was used in the present study prevented ulceration and enhanced healing in a model of alkali-induced corneal injury (11). In this study we evaluated the effect of inhibition of MMP activity in EAE by administration of a hydroxamate MMP inhibitor (GM 6001).

\section{Methods}

EAE induction. Female SJL/J mice (Jackson Laboratory, Bar Harbor, $\mathrm{ME}$ ) were used at 5-7 wk of age. EAE was actively induced by subcutaneous immunization with $200 \mu \mathrm{g}$ of the encephalitogenic proteolipid protein peptide, PLP aa139-151, in CFA plus $0.2 \mathrm{mg}$ Mycobacterium tuberculosis H37RA (Difco Laboratories, Inc., Detroit, MI). PLP peptide 139-151 was prepared by continuous flow solid phase synthesis according to the sequence HCLGKWLGHPDKF by the Protein and Nucleic Acid Facility, Beckman Center, Stanford, CA. Peptide purity 


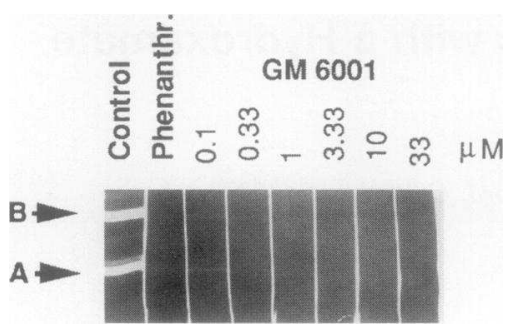

Figure 1. Inhibition of CSF gelatinase activity by GM 6001. Pooled CSF from animals with EAE was run on an SDSPAGE gel containing gelatin. After electrophoresis the gel was cut into strips and these were incubated for development of the enzymatic activity with various concentrations of the inhibitor. Gelatinase A and B were both present in the CSF and were equally well inhibited. GM 6001 completely inhibited all gelatinase activity at a concentration of $1 \mu \mathrm{M}$ and above; there was partial inhibition at 0.1 and $0.33 \mu \mathrm{M}$. The metalloprotease inhibitor phenanthroline $(2.5 \mathrm{mM})$ served as an inhibitory control.

was examined by HPLC analysis and peptide identity was confirmed by amino-acid composition analysis.

Clinical scoring of EAE. EAE was scored clinically as follows: no neurological signs $=$ grade 0 , weak tail $=$ grade 1 , wobbly walk or limb paresis $=$ grade 2 , limb paralysis $=$ grade 3 , inability to move $=$ grade 4 , death $=$ grade 5 . Easy access to food and water was provided and animals with grade 3 or 4 received daily parenteral fluid.

Metalloprotease inhibitor and treatment. The peptide hydroxamic acid, $N$-[2(R)-2-(hydroxamido carbonylmethyl)-4-methylpentanoyl]L-tryptophane methylamide (GM 6001; Glycomed Inc., Alameda, CA), (12) was dissolved in $4 \%(\mathrm{wt} / \mathrm{vol})$ carboxymethylcellulose in water and administered by $0.2 \mathrm{ml}$ intraperitoneal injection. The same volume of vehicle was used as a control treatment. Animals were treated daily either from the day of disease induction for $20 \mathrm{~d}$ or from the onset of clinical signs for $10 \mathrm{~d}$ (randomized for the different treatment groups).

Blood-brain barrier permeability. Mice were injected intravenously with $1 \%$ (wt/vol) Evans blue (Fisher Scientific Co., Pittsburgh, PA) in PBS at a dosage of $4 \mu \mathrm{l} / \mathrm{g}$ body $\mathrm{w}$ (13). After $1 \mathrm{~h}$ the animals were euthanized by $\mathrm{CO}_{2}$ inhalation and perfused with PBS via the left ventricle. Brain and spinal cord were dissected and cut longitudinally. Half of the CNS was used for histopathological evaluation. The other half was weighed and tissue samples were then extracted for $3 \mathrm{~d}$ in formamide ( $5 \mu \mathrm{l} / \mathrm{mg}$ tissue) and the extracts centrifuged for $5 \mathrm{~min}$ at $500 \mathrm{~g}$. Evans blue concentration in the extracts was determined by measuring the absorbance at $650 \mathrm{~nm}$. The blood-brain barrier permeability index was calculated by dividing the value for each sample by the corresponding (brain or spinal cord) mean value of four normal noninduced animals.

Histopathology. Brain and spinal cord were fixed for $24 \mathrm{~h}$ in $4 \%$ (wt/vol) paraformaldehyde in PBS, whereafter they were paraffin embedded and sectioned. Sections were stained with hematoxylin and eosin, Klüver-Barrera and Giemsa stain. Histopathology was assessed as follows. Individual inflammatory lesions were scored as $1=$ perivascular inflammatory cuff $\leq$ three cells thick, $2=>$ three cells thick, $3=$ parenchymal infiltrate. The total inflammatory score was then calculated by adding all the scores for individual lesions from all the sections for a given sample. The demyelination score was calculated the same way as inflammatory score, with $1=$ demyelinated rim around infiltrate, 2 $=$ demyelination extending into noninfiltrated tissue.

In vivo inhibitor kinetics. Normal noninduced animals or animals with clinical signs of EAE for $2 \mathrm{~d}$ were given a single intraperitoneal injection of $4 \mathrm{mg} \mathrm{GM} 6001$ and euthanized by $\mathrm{CO}_{2}$ inhalation after various time intervals. CSF was taken by suboccipital puncture as described previously (14). Pooled CSF from five animals per time point was frozen immediately at $-70^{\circ} \mathrm{C}$. GM 6001 concentrations were determined by reversed-phase HPLC by Bay Bioanalytical Laboratories, Richmond, CA. CSF was diluted into $20 \%$ acetonitrile in water and chromatographed on a C-18 reversed-phase column with fluorescence detection. Linearity was determined by spiking blank CSF with increasing amounts of GM 6001. The amount of GM 6001 in CSF samples

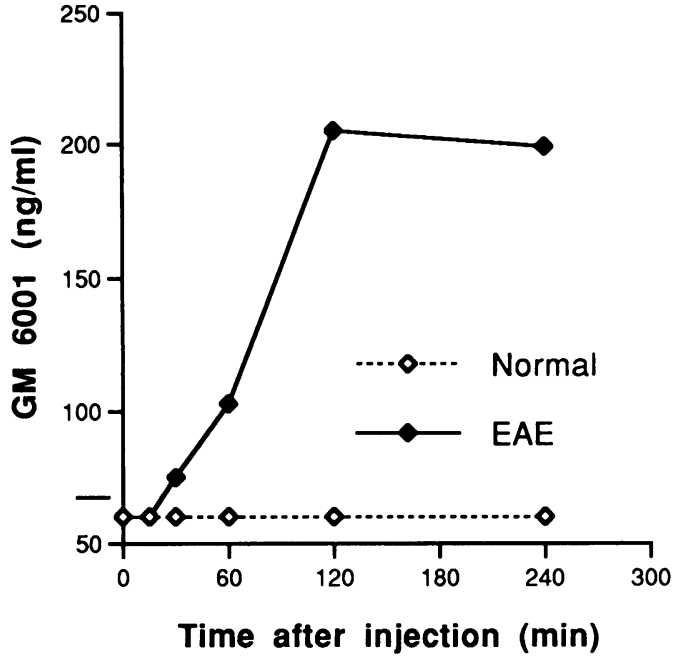

Figure 2. GM 6001 levels in the CSF after a single intraperitoneal injection. Animals with EAE or noninduced normal animals received 4 mg GM 6001 intraperitoneally. They were killed after various time intervals and CSF was taken and pooled (five animals/time point). GM 6001 concentrations were determined by reversed-phase HPLC. Levels stayed under the detection limit $(60 \mathrm{ng} / \mathrm{ml})$ in normal animals, but in animals with EAE they were detectable $30 \mathrm{~min}$ after injection and were in the $100-200 \mathrm{ng} / \mathrm{ml}$ range $(\sim 0.25-0.50 \mu \mathrm{M})$ for at least $3 \mathrm{~h}$.

was calculated based on recovery of GM 6001 and the internal standard in spiked samples. The lower limit for quantification of GM 6001 in the CSF samples was $60 \mathrm{ng} / \mathrm{ml}$.

In vitro inhibition of gelatinase activity. Gelatinase activity in a pooled CSF sample from animals with clinical signs of EAE (grade 23 for 4-5 d) was determined by SDS/PAGE zymography. CSF was applied without prior denaturation to a single-well $7.5 \%$ (wt/vol) polyacrylamide gel containing $0.1 \%$ ( wt $/ \mathrm{vol}$ ) SDS to which $0.1 \%(\mathrm{wt} / \mathrm{vol}$ ) tissue culture grade gelatin (Sigma Chemical Co., St. Louis, MO) was added and copolymerised. The stacking gel was $5 \%(\mathrm{wt} / \mathrm{vol})$ polyacrylamide and did not contain gelatin. The gel $(7 \mathrm{~cm} \times 10 \mathrm{~cm} \times 0.75 \mathrm{~mm})$ was run for approximately $2 \mathrm{~h}$ at $20 \mathrm{~mA}$. After electophoresis the gel was washed (washing buffer: $50 \mathrm{mM}$ Tris- $\mathrm{HCl}, 10 \mathrm{mM} \mathrm{CaCl}_{2}, 0.02 \%$ (wt/vol) $\mathrm{NaN}_{3}, 2.5 \%$ ( vol/vol) Triton X-100, $\mathrm{pH} \mathrm{7.5)} \mathrm{to} \mathrm{remove} \mathrm{the}$ SDS and sliced into strips. The resulting strips were incubated separately at $37^{\circ} \mathrm{C}$ during $24 \mathrm{~h}$ (same buffer as for the washing, containing only $1 \%$ [ $\mathrm{vol} / \mathrm{vol}]$ Triton X-100) for development of the enzyme activity, stained with Coomassie brilliant blue R-250 and destained in methanol/ acetic acid. Gelatinase activity was detected as unstained bands on a blue background. To assess the effect of protease inhibitors on the gelatinase activity present in the CSF, metalloprotease inhibitors (dissolved in DMSO) were added to the incubation buffer. GM 6001 was used at final concentrations from 0.1 to $33 \mu \mathrm{M} ; 1,10$-phenantroline (Sigma Chemical Co.) was used as a positive control at $2.5 \mathrm{mM}$, and the noninhibitor lane was incubated in $0.5 \%$ ( vol $/ \mathrm{vol})$ DMSO.

Statistics. The nonparametric Mann-Whitney U-test was used.

\section{Results}

Fig. 1 shows the in vitro effect of GM 6001 on gelatinase activity in the CSF from animals with EAE. Both gelatinase A and $B$ were inhibited to the same extent by GM 6001. Concentrations of the inhibitor from 33 to $1 \mu \mathrm{M}$ were completely inhibitory, and the lowest concentration tested, $0.1 \mu \mathrm{M}$, was still partially effective.

A single intraperitoneal dose of $4 \mathrm{mg}$ GM 6001 was followed by detectable levels of GM 6001 in the CSF of animals 


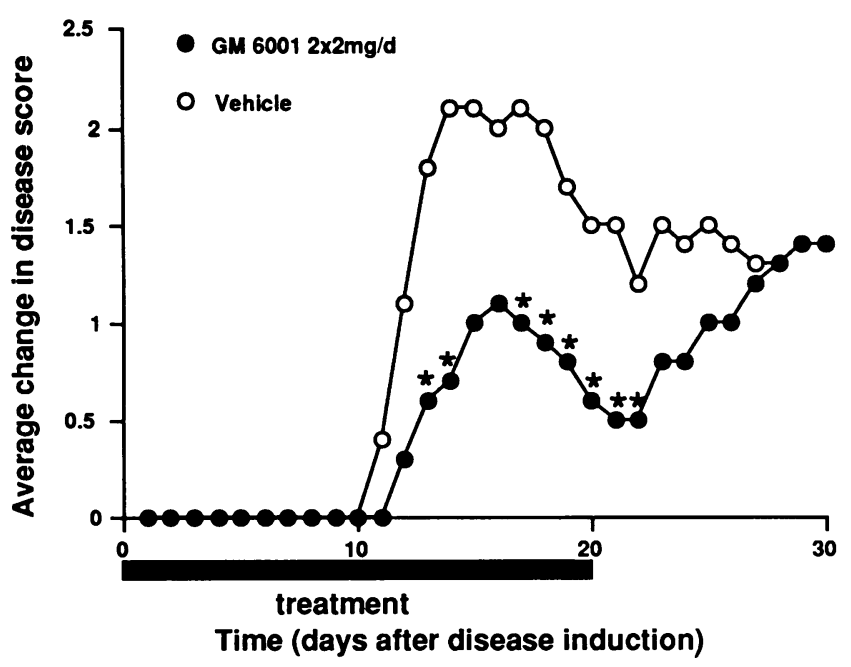

Figure 3. Preventive effect of GM 6001 on the development of EAE. Animals were treated from disease induction for $20 \mathrm{~d}$ with vehicle or GM $60012 \times 2 \mathrm{mg} / \mathrm{d}$ intraperitoneally. Clinical signs were scored as described in Methods. The average change in disease score (relative to the first day of treatment) is given for 10 animals in each treatment group. The clinical severity of the disease was significantly reduced in the GM 6001-treated group compared with the vehicle-treated group. The differences between the treatment groups became insignificant $2 \mathrm{~d}$ after cessation of treatment ( $* P<0.05$, Mann-Whitney U-test) (experiment 1 in Table I). with EAE, starting 30 min after injection for at least $4 \mathrm{~h}$ (Fig. 2 ). Concentrations were between 100 and $200 \mathrm{ng} / \mathrm{ml}$ for at least $3 \mathrm{~h}$, which is in the $0.25-0.50 \mu \mathrm{M}$ range. In contrast, GM 6001 concentrations in the CSF of normal noninduced animals never rose above the detection limit after the same dose of the inhibitor.

Two different treatment schedules were used: $(a)$ treatment from disease induction (to evaluate a possible preventive effect ), ( $b$ ) treatment from the onset of clinical signs (to evaluate a possible disease reversing effect). Animals that were treated with vehicle showed the typical clinical course of our EAE model. Clinical signs compatible with an ascending myelitis started from day 10-12 after induction and disease peaked around day 14-17, whereafter a variable degree of recovery followed (Fig. 3 ). Several animals went into a clinical relapse from day 20 onwards, after previous partial or complete recovery. Treatment with $2 \times 2 \mathrm{mg} \mathrm{GM} 6001$ daily from disease induction delayed the onset of the disease and significantly reduced clinical disease severity (Table I and Fig. 3) $(P$ $<0.05)$. A significant reduction in disease score in the GM 6001 -treated group compared with the vehicle-treated group persisted for $2 \mathrm{~d}$ after cessation of treatment, whereafter the average disease score of the two groups became similar.

In the second part of this experiment, previously untreated animals were randomly assigned at the onset of clinical signs to be treated either with GM $60012 \times 2 \mathrm{mg} / \mathrm{d}$ or with vehicle twice a day, for $10 \mathrm{~d}$. In animals treated with vehicle, clinical severity increased for a few days, whereafter the animals slowly recovered (Table I and Fig. 4). In contrast, clinical scores in animals treated with GM 6001 decreased the day after the start

Table I. Clinical effect of GM 6001 on EAE

\begin{tabular}{|c|c|c|c|c|c|c|}
\hline \multirow{2}{*}{$\begin{array}{l}\text { Group } \\
\text { treatment schedule }\end{array}$} & \multirow{2}{*}{\multicolumn{2}{|c|}{ Treatment }} & \multirow[b]{2}{*}{ Dose } & & \multicolumn{2}{|c|}{$\begin{array}{l}\text { Change in average cumulative disease } \\
\text { score* }\end{array}$} \\
\hline & & & & & During treatment & After treatment \\
\hline \multicolumn{7}{|l|}{ Experiment $1^{\S}$} \\
\hline \multirow[t]{2}{*}{ From disease induction for $20 \mathrm{~d}$} & Vehicle & $2 \times / d$ & - & 10 & $16.8 \pm 2.06$ & $13.9 \pm 3.91$ \\
\hline & GM 6001 & $2 \times / d$ & 2.00 & 10 & $7.0 \pm 2.57^{\natural}$ & $9.9 \pm 2.17$ \\
\hline \multirow[t]{2}{*}{ From onset of clinical signs for $10 \mathrm{~d}$} & Vehicle & $2 \times / d$ & - & 10 & $5.0 \pm 1.99$ & $-2.7 \pm 5.53$ \\
\hline & GM 6001 & $2 \times / d$ & 2.00 & 10 & $-6.5 \pm 2.08^{* *}$ & $-7.2 \pm 2.69$ \\
\hline \multicolumn{7}{|l|}{ Experiment 2} \\
\hline \multirow[t]{5}{*}{ From disease induction for $20 \mathrm{~d}$} & Vehicle & $1 \times / d$ & - & 10 & $14.7 \pm 1.80$ & $20.7 \pm 6.06$ \\
\hline & GM 6001 & $1 \times / d$ & 4.00 & 10 & $2.4 \pm 1.16^{* *}$ & $22.2 \pm 4.61$ \\
\hline & & $1 \times / d$ & 1.33 & 10 & $3.4 \pm 1.77 * *$ & $12.0 \pm 3.83$ \\
\hline & & $1 \times / d$ & 0.40 & 10 & $11.3 \pm 0.94$ & $23.9 \pm 4.96$ \\
\hline & & $1 \times / d$ & 0.13 & 10 & $7.7 \pm 3.09$ & $18.0 \pm 9.82$ \\
\hline \multirow[t]{5}{*}{ From onset of clinical signs for $10 \mathrm{~d}$} & Vehicle & $1 \times / d$ & - & 8 & $5.1 \pm 2.55$ & $6.5 \pm 10.4$ \\
\hline & GM 6001 & $1 \times / d$ & 4.00 & 9 & $-4.3 \pm 2.69$ & $-7.1 \pm 7.86$ \\
\hline & & $1 \times / d$ & 1.33 & 9 & $-4.1 \pm 1.81^{\uparrow}$ & $-15.0 \pm 5.79$ \\
\hline & & $1 \times / d$ & 0.40 & 9 & $-0.3 \pm 2.03$ & $-7.9 \pm 4.44$ \\
\hline & & $1 \times / d$ & 0.13 & 9 & $2.0 \pm 1.92$ & $-10.6 \pm 5.67$ \\
\hline
\end{tabular}

For clinical scoring, see Methods. * Change relative to the clinical score at the onset of treatment (mean disease score at the onset of clinical signs for the groups treated from this moment: experiment 1, 1.50 [vehicle] and 1.60 [GM 6001]; experiment 2, 1.63 [vehicle], 1.78 [GM $6001,4 \mathrm{mg}$ / d], 1.78 [GM 6001, $1.33 \mathrm{mg} / \mathrm{d}$ ], 1.89 [GM 6001, $0.4 \mathrm{mg} / \mathrm{d}$ ], 1.56 [GM 6001, $0.13 \mathrm{mg} / \mathrm{d}$ ]). ${ }^{\ddagger}$ Follow-up 10 (experiment 1) or 20 d (experiment 2 ). "Time course depicted in Figs. 3 and 4 . "Average values \pm SEM. " $P<0.05$. $* * P<0.005$ compared with vehicle-treated animals (MannWhitney U-test). 


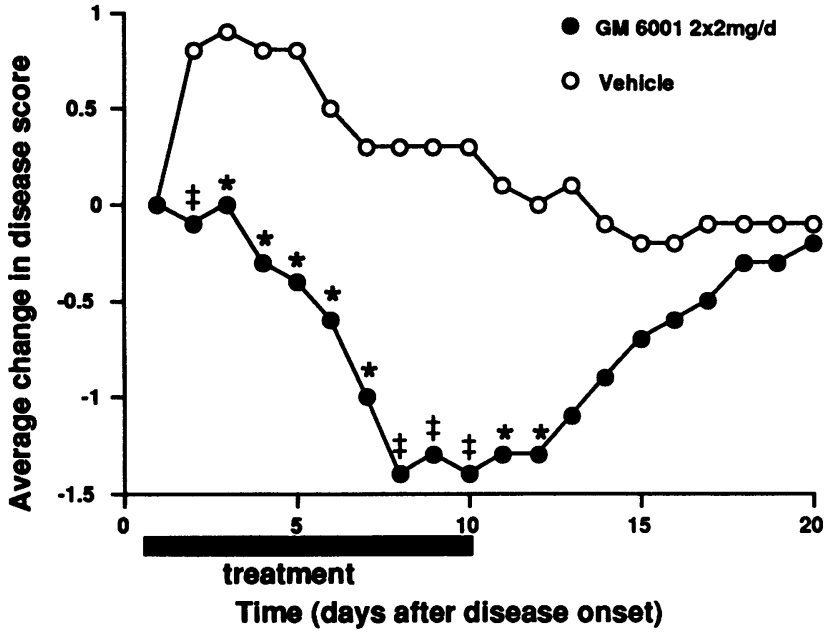

Figure 4. Reversal of EAE by GM 6001. Animals were treated from the onset of clinical signs for $10 \mathrm{~d}$ with vehicle or GM $60012 \times 2 \mathrm{mg} /$ $\mathrm{d}$ intraperitoneally. Clinical signs were scored as described in Methods section. The average change in disease score (relative to the first day of treatment) is given for 10 animals in each treatment group. The clinical severity of the disease was significantly reduced in the GM 6001 treated group compared to the vehicle treated group. The differences between the treatment groups became insignificant $2 \mathrm{~d}$ after cessation of treatment $\left({ }^{*} P<0.05 ;{ }^{\ddagger} P<0.005\right.$, Mann-Whitney U-test $)$ (Experiment 1 in Table I).

of treatment and this difference with the vehicle-treated group remained statistically significant for up to two days after treatment $(P<0.05) .8$ out of 10 animals treated with GM 6001 returned to score 0 (clinically normal) within the $12 \mathrm{~d}$ following the onset of clinical signs (compared with 3 out of 10 treated with vehicle), 7 of these animals had a clinical disease relapse during the follow-up period (compared with all 3 treated with vehicle).

In a second experiment, the same treatment schedules were used, but with only one intraperitoneal injection daily and with various doses of the inhibitor. A single daily dose of $4 \mathrm{mg} / \mathrm{d}$ had a comparable efficacy as $2 \times 2 \mathrm{mg} / \mathrm{d}$ in both treatment schedules (Table I). Doses as low as $0.13 \mathrm{mg} / \mathrm{d}$ were still effective if given from disease induction $(4$ and $1.33 \mathrm{mg} / \mathrm{d}, P$ $<0.005 ; 0.13 \mathrm{mg} / \mathrm{d}, P<0.05)$. When treated from disease onset, there was a clear dose-effect relationship; only 4 and $1.33 \mathrm{mg} / \mathrm{d}$ were significantly effective in reducing disease severity $(P<0.05)$. Animals treated with GM 6001 recovered faster from the drop in body weight that is associated with the onset of EAE, but the differences between the GM 6001 treated groups and the vehicle-treated group were never statistically significant (Fig. 5). (It should be noted that animals received parenteral fluid therapy, once they reached a grade 3 clinical score )

The degree of inflammation and demyelination was compared histopathologically for animals treated with GM 6001 (1 $\times 1.33 \mathrm{mg} / \mathrm{d}$ ) or with vehicle from the onset of clinical signs (Table II). The inflammatory and demyelination scores were lower after 3 and $10 \mathrm{~d}$ of treatment with GM 6001 in all but one group, but the differences did not reach statistical significance. The blood-brain barrier index, however, was significantly elevated after $10 \mathrm{~d}$ of disease in the vehicle-treated animals but was normal in the GM 6001 group $(P<0.05)$.

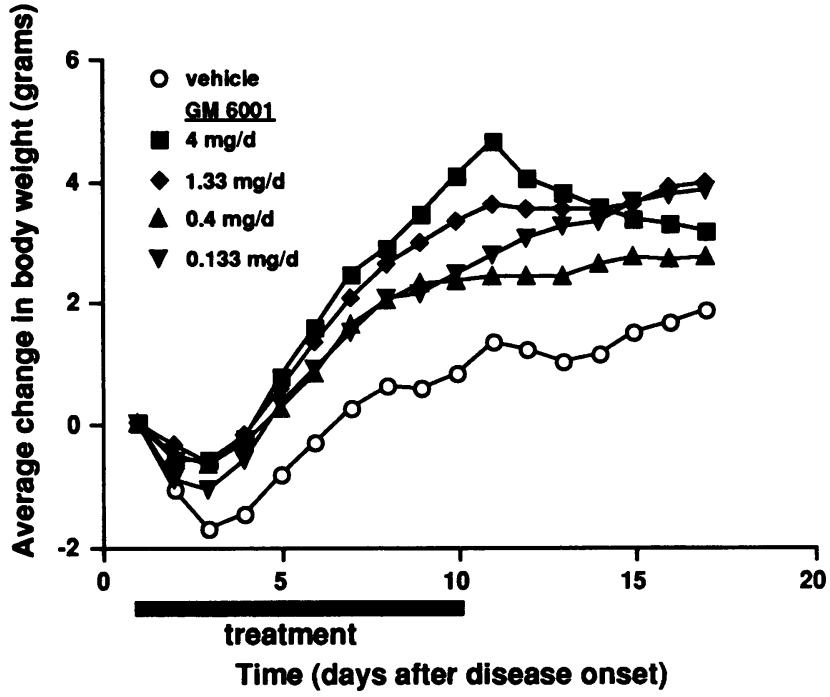

Figure 5. Effect of GM 6001 on weight loss in EAE. Animals were treated from the onset of clinical signs for $10 \mathrm{~d}$ with vehicle or GM 6001 in doses from 4 to $0.13 \mathrm{mg} / \mathrm{d}$ intraperitoneally. Body weight was measured and recorded daily from the onset of clinical signs. The average change in body weight relative to the body weight at the onset of clinical signs is shown for the different treatment groups. The differences between the GM 6001-treated groups and the vehicle-treated group were not significant at any moment in time (Mann-Whitney U-test) (experiment 2 in Table I, $n=8-9$ animals/group).

\section{Discussion}

Since MMPs appear to play an important role in lesion development in inflammatory demyelinating diseases such as MS, inhibition of the activity of these enzymes might offer a new therapeutic approach in these disorders. We demonstrated that administration of the MMP inhibitor GM 6001 not only suppressed the development of EAE, but was also able to reverse ongoing disease in a dose-dependent way (Table I; Figs. 3 and 4). A single parenteral dose of the inhibitor given to animals with EAE caused levels in the CSF, sufficient to induce at least a partial inhibition of the gelatinase activity in the CSF (Figs. 1 and 2). It should be noted that the assay used to determine inhibitor activity on zymography probably underestimates the inhibiting capacity because of the time necessary for the inhibitor to diffuse into the gel; during this interval some enzyme activity could already develop. Based upon these findings, i.e., timing and CNS penetration, we conclude that the inhibitor is acting at the level of the CNS lesion.

Disease development and spontaneous recovery in this model are too fast to be caused by de- and remyelination, respectively. Thus the rapid effect of the protease inhibitor used in this study could probably not be caused by an inhibition of demyelination. This was confirmed by histopathological studies that showed only a moderate decrease in demyelination and inflammation in animals treated from the onset of clinical signs (Table II). However, the blood-brain barrier, which was significantly impaired in vehicle-treated animals, was restored in animals treated with the MMP inhibitor. Increased blood-brain barrier permeability with subsequent CNS edema and entry of inflammatory mediators such as complement and antibodies (15) could lead to neurological dysfunction by affecting axonal conduction even before demyelination occurs. The fact that the 
Table II. Effect of GM 6001 blood-brain barrier permeability and pathology in EAE

\begin{tabular}{|c|c|c|c|c|c|c|}
\hline \multirow[b]{2}{*}{ Treatment group } & & \multicolumn{2}{|c|}{ Blood-brain barrier index* } & \multicolumn{2}{|c|}{ Inflammation ${ }^{\ddagger}$} & \multirow{2}{*}{$\frac{\text { Demyelination }^{\S}}{\text { Brain }+ \text { spinal cord }}$} \\
\hline & & Brain & Spinal cord & Brain & Spinal cord & \\
\hline & $n$ & & & & & \\
\hline \multicolumn{7}{|l|}{$3 \mathrm{~d}$} \\
\hline Vehicle & 4 & $1.2 \pm 0.08$ & $1.0 \pm 0.14$ & $47.2 \pm 12.3$ & $29.0 \pm 9.26$ & $7.0 \pm 1.68$ \\
\hline GM $6001^{\pi}$ & 3 & $1.1 \pm 0.16$ & $0.7 \pm 0.12$ & $31.7 \pm 5.17$ & $14.7 \pm 1.67$ & $3.7 \pm 0.88$ \\
\hline \multicolumn{7}{|l|}{$10 \mathrm{~d}$} \\
\hline Vehicle & 4 & $1.8 \pm 0.29$ & $1.6 \pm 0.07$ & $26.8 \pm 1.25$ & $14.5 \pm 1.50$ & $4.5 \pm 1.50$ \\
\hline GM 6001 & 4 & $0.8 \pm 0.07 * *$ & $1.1 \pm 0.11^{* *}$ & $21.0 \pm 3.14$ & $15.0 \pm 3.24$ & $2.3 \pm 0.63$ \\
\hline
\end{tabular}

* Ratio of permeability for Evan's blue; ${ }^{\ddagger}$ degree of inflammation or ${ }^{\star}$ demyelination, calculated as described in Methods; " average values \pm SEM;

$" 1 \times 1.33 \mathrm{mg} / \mathrm{d}$ intraperitoneally; ${ }^{* *} P<0.05$ compared with vehicle-treated animals (Mann-Whitney U-test).

clinical disease course became essentially the same in inhibitortreated as in vehicle-treated animals after cessation of treatment is another indication that this treatment is affecting the inflammatory reaction (rather than the immune response against the myelin sheath that is initiating and perpetuating the inflammatory reaction).

The same considerations are true for the major human inflammatory demyelinating disease of the CNS, multiple sclerosis, where clinical relapses and remissions correlate with inflammatory activity and breakdown of the blood-brain barrier rather than with demyelination $(16,17)$. Demyelination, however, is thought to be a consequence of the inflammatory reaction and is the cause of the chronic neurological deficit associated with this disease by impairing axonal conduction (18). Inhibition of the initial inflammatory reaction and the associated perturbation of the blood-brain barrier is thus not only important for the treatment of the acute attack, but could also prevent subsequent demyelination.

Presently, we only have evidence for the involvement of gelatinases in inflammatory demyelinating processes $(4,5,8$, 9 ). However, other MMPs could also play a role in this tissuedestructive process since these enzymes are known to act in concert (6). The MMP inhibitor used in the present study is not specific for one of the MMPs (GM 6001 has a $K_{\mathrm{i}}$ of 0.4 $\mathrm{nM}$ against fibroblast collagenase, $0.5 \mathrm{nM}$ against gelatinase $\mathrm{A}$, $0.2 \mathrm{nM}$ against gelatinase $\mathrm{B}, 0.1 \mathrm{nM}$ against neutrophil collagenase, and $27 \mathrm{nM}$ against stromelysin; in contrast to a $K_{\mathrm{i}}$ of 0.3 $\mathrm{mM}$ against the metalloprotease angiotensin-converting enzyme) (19, and unpublished results). The effect demonstrated is thus not informative about the role of a single MMP (in this case gelatinase) in EAE. The use of more specific MMP inhibitors can help to solve this question.

Several studies have shown a suppressive effect of various protease inhibitors on the development of EAE. The effect of inhibitors that interfere with the plasminogen/plasmin system $(20-23)$ could be explained by the in vitro findings that this system controls MMP activation (24). Inhibition of the lysosomal enzyme cathepsin D interferes with antigen processing and probably suppresses EAE by downregulating the immune response against the antigenic inoculum used for EAE induction (25). The present study is the first to our knowledge where disease that was already clinically apparent could be suppressed. Since inflammatory lesions are present in the CNS in EAE even a few days before the onset of clinical signs (5), our results indicate that GM 6001 is effective during the inflammatory phase of the disease.

Although demyelination is only moderate in EAE as compared to MS, we think that EAE as used in this study is an appropriate model for evaluation of therapeutic intervention in a single MS relapse. Long-term studies in which the MMP inhibitor is administered chronically or where recurrent EAE relapses are treated will probably be more informative regarding the effect on demyelination and chronic disease. New therapeutic approaches for MS and EAE have been reviewed extensively $(2,26,27)$. The inhibition of matrix metalloproteases might be used in the future in concert with more selective therapies for demyelinating disease which target the ternary complex of $\mathrm{T}$ cell receptor, $\mathrm{MHC}$, and antigen.

\section{Acknowledgment}

Work in the authors' laboratory was supported by the National Institutes of Health.

\section{References}

1. Allen, I. V. 1989. Pathology of multiple sclerosis. In McAlpine's multiple sclerosis. Second edition. W. B. Matthews, A. Compston, I. V. Allen, and C. N. Martyn, editors. Churchill Livingstone, Edinburgh. 341-378.

2. Steinman, L., A. Miller, C. C. A. Bernard, and J. R. Oksenberg. 1994. The epigenetics of multiple sclerosis: clues to etiology and a rationale for immune therapy. Annu. Rev. Neurosci. 17:247-265.

3. Banik, N. L. 1992. Pathogenesis of myelin breakdown in demyelinating diseases: role of proteolytic enzymes. Crit. Rev. Neurobiol. 16:257-271.

4. Gijbels, K., S. Masure, H. Carton, and G. Opdenakker. 1992. Gelatinase in the cerebrospinal fluid of patients with multiple sclerosis and other inflammatory neurological disorders. J. Neuroimmunol. 41:29-34.

5. Gijbels, K., P. Proost, S. Masure, H. Carton, A. Billiau, and G. Opdenakker. 1993. Gelatinase B is present in the cerebrospinal fluid during experimental autoimmune encephalomyelitis and cleaves myelin basic protein. J. Neurosci. Res. 36:432-440.

6. Kleiner, D. E., Jr., and W. G. Stetler-Stevenson. 1993. Structural biochemistry and activation of matrix metalloproteases. Curr. Opin. Cell Biol. 5:891-897.

7. Mackay, A. R., J. L. Hartzler, M. D. Pelina, and U. P. Thorgeirsson. 1990. Studies on the ability of $65-\mathrm{kDa}$ and $92-\mathrm{kDa}$ tumor cell gelatinases to degrade type IV collagen. J. Biol. Chem. 265:21929-21934.

8. Rosenberg, G. A., M. Kornfeld, E. Estrada, R. O. Kelley, L. A. Liotta, and W. G. Stetler-Stevenson. 1992. TIMP-2 reduces proteolytic opening of the bloodbrain barrier by type IV collagenase. Brain Res. 576:203-207.

9. Gijbels, K., and L. Steinman. 1994. Gelatinase B producing cells in multiple sclerosis lesions. J. Cell. Biochem. Suppl. 18D:143.

10. DiMartino, M. J., C. E. Wolff, W. High, M. Crimmin, and W. A. Galloway. 1991. Antiinflammatory and chondroprotective activities of a potent metalloproteinase inhibitor. J. Cell. Biochem. Suppl. 19E:179. 
11. Schultz, G. S., S. Strelow, G. A. Stern, N. Chegini, M. B. Grant, R. E. Galardy, D. Grobelny, J. J. Rowsey, K. Stonecipher, V. Parmley, and P. T. Khaw. 1992. Treatment of alkali-injured rabbit corneas with a synthetic inhibitor of matrix metalloproteinases. Invest. Opthalmol. \& Visual Sci. 33:3325-3331.

12. Grobelny, D., L. Poncz, and R. E. Galardy. 1992. Inhibition of human skin fibroblast collagenase, thermolysin, and Pseudomonas aeruginosa elastase by peptide hydroxamic acids. Biochemistry. 31:7152-7154.

13. Farrell, C. L., P. A. Stewart, and R. F. Del Maestro. 1987. A new glioma model in rat: the $\mathrm{C} 6$ spheroid implantation technique permeability and vascular characterization. J. Neuro-Oncol. 4:403-415.

14. Gijbels, K., P. Proost, W. Put, J. Van Damme, and A. Billiau. 1990 Interleukin 6 production in the central nervous system during experimental autoimmune encephalomyelitis. Eur. J. Immunol. 20:233-235.

15. Rasminsky, M. 1984. Pathophysiology of demyelination. Ann. NY Acad. Sci. 436:68-85.

16. McDonald, W. I., D. H. Miller, and D. Barnes. 1992. The pathological evolution of multiple sclerosis. Neuropathol. Appl. Neurobiol. 18:319-334.

17. Youl, B. D., G. Turano, D. H. Miller, A. D. Twell, D. G. MacManus, S. G. Moore, S. J. Jones, G. Barrett, B. E. Kendall, I. F. Moseley, et al. 1991. The pathophysiology of acute optic neuritis. An association of gadolinium leakage with clinical and electrophysiological deficits. Brain. 114:2437-2450.

18. Waxman, S. G. 1988. Clinical course and electrophysiology of multiple sclerosis. Adv. Neurol. 47:157-184.
19. Galardy, R. E., M. E. Casabonne, C. Giese, J. H. Gilbert, F Lapierre, H Lopez, M. E. Schaefer, R. Stack, M. Sullivan, B. Summers, et al. 1994. Low molecular weight inhibitors in corneal ulceration. Ann. NY Acad. Sci. In press.

20. Sibley, W. A., J. Kiernat, and J. F. Laguna. 1978. The modification of experimental allergic encephalomyelitis with epsilon aminocaproic acid. Neurology. 28:102-105.

21. Brosnan, C. F., W. Cammer, W. T. Norton, and B. M. Bloom. 1980 Proteinase inhibitors suppress the development of experimental allergic encephalomyelitis. Nature (Lond.). 285:235-237.

22. Smith, M. E., and L. A. Amaducci. 1982. Observations on the effects of protease inhibitors on the suppression of experimental allergic encephalomyelitis Neurochem. Res. 7:541-554.

23. Inuzuka, T., S. Sato, H. Baba, and T. Miyatake. 1988. Suppressive effect of camostat mesilate (FOY 305 ) on acute experimental allergic encephalomyelitis (EAE). Neurochem. Res. 13:225-228.

24. Murphy, G., S. Atkinson, R. Ward, J. Gavrilovic, and J. J. Reynolds 1992. The role of plasminogen activators in the regulation of connective tissue metalloproteinases. Ann. NY Acad. Sci. 667:1-12.

25. Boehme, D. H., H. Umezawa, G. Hashim, and N. Marks. 1978. Treatment of experimental allergic encephalomyelitis with an inhibitor of cathepsin D (pepstatin ). Neurochem. Res. 3:185-194.

26. Steinman, L. 1991. The development of rationale strategies for selective immunotherapy against demyelinating disease. Adv. Immunol. 49:357-379.

27. Steinman, L. 1993. Autoimmune disease. Sci. Am. 269:106-114. 